\title{
Treatment of intermittent intussusception in Peutz- Jeghers syndrome with single-balloon enteroscopy
}
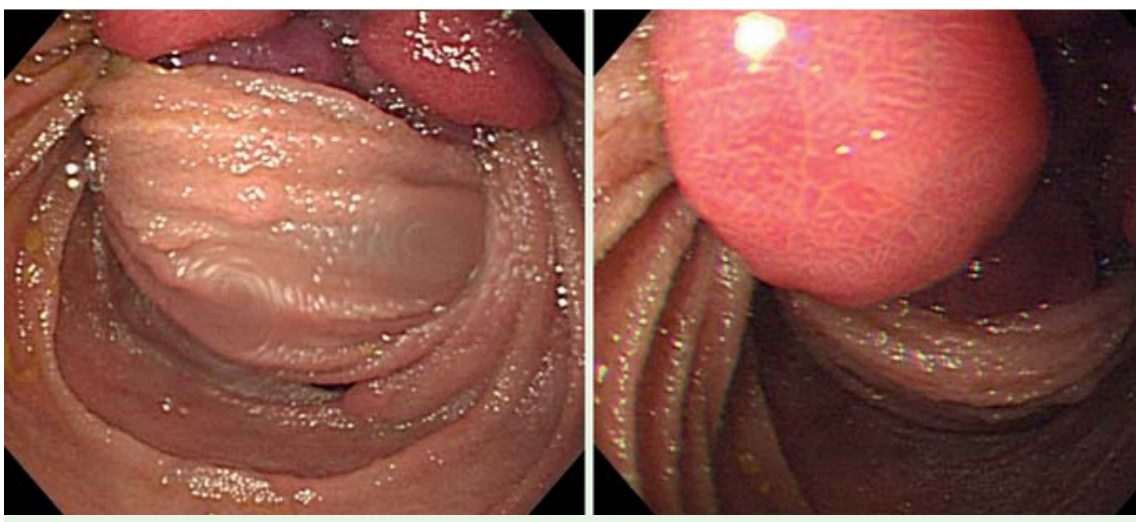

Fig. 1 Transient intussusception in the proximal jejunum caused by a large polyp.

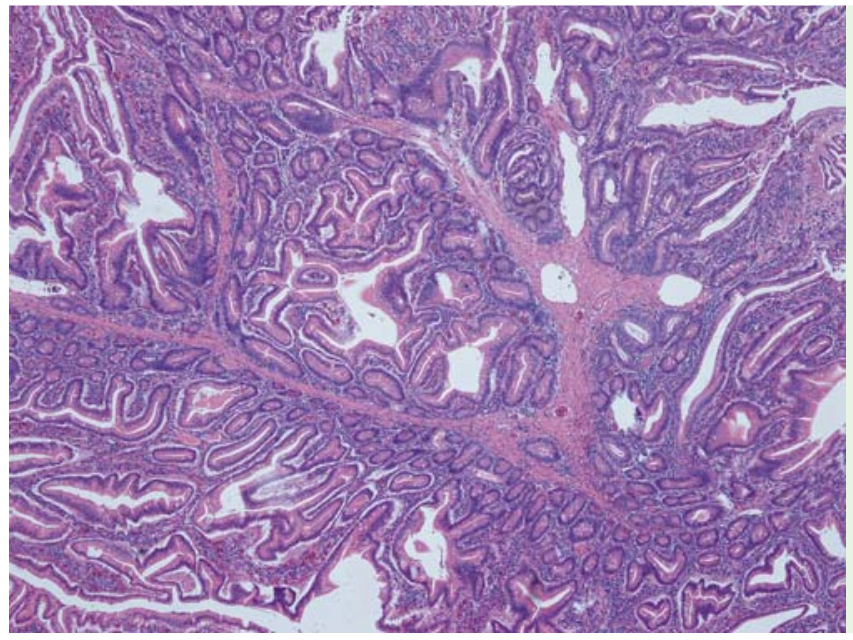

Fig. 3 Histopathological examination showed a hamartomatous polyp.

A 30-year-old woman with Peutz-Jeghers syndrome (PJS) had a known history of jejunal intussusception with partial resection of the jejunum 7 years previously. The PJS was under surveillance with regular polypectomy by colonoscopy every year in our hospital. She attended our department again with a 1 month history of intermittent upper abdominal cramping pain and bile-like vomitus. Single-balloon enteroscopy (SBE) showed transient intussusception in the proximal jejunum which was caused by a 3-cm broad-based polyp ( Fig. 1).

The intussusception was successfully reduced by inflating the jejunum using SBE. The single-balloon enteroscope was advanced to the distal jejunum where more than 10 polyps were also found. Polyps larger than $0.5 \mathrm{~cm}$ were resected. Total resection of the leading point of the intussusception, the broad-based polyp, was difficult, so partial polypectomy was done to prevent the risk of intussusception again ( $\bullet$ Fig. 2 ).

We suggested surgical intervention, but patient refused because the symptoms had been relieved.

The patient continued well until 3 months after the procedure. She developed lower abdominal pain gradually, and abdominal computed tomography revealed a mass measuring $14.3 \times 8.3 \mathrm{~cm}$ mass in the pelvic cavity. Explorative laparotomy revealed an ovarian teratoma. The residual broadbased polyp was also resected during laparotomy. Fifteen polyps (about $0.5-2 \mathrm{~cm}$ in size) in the jejunum and ileum were

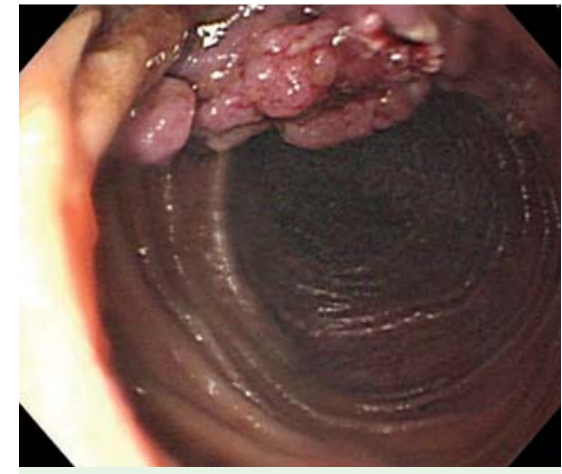

Fig. 2 The broad-based polyp after partial polypectomy.

removed under intraoperative enteroscopy. Pathological analysis of these polyps showed hamartomatous polyps ( $\mathbf{F i g}$. 3 ). PJS is characterized by multiple hamartomatous polyps throughout the entire gastrointestinal tract, most commonly in the small intestine. The most common symptoms of PJS in the small bowel are obstruction and/or intussusception [1]. Surveillance of PJS with regular endoscopic polypectomy to prevent the bowel loss resulting from emergent resection was suggested in the past. The doubleballoon enteroscopy (DBE) system was presented for the first time in 2001, and now we can perform small intestine evaluation and polypectomy for PJS more easily [2,3]. Another mode of balloon-assisted enteroscopy using a single balloon is a new technique (SBE) which is safe and easier to learn than DBE [4].

In our patient, the preceding surveillance of PJS with colonoscopy was inadequate. If balloon-assisted enteroscopy had been performed after the previous surgery 7 years ago, the large broad-based polyp might have been removed when it was relatively small. When polyps are in the small bowel and difficult to resection totally by enteroscopy, partial polypectomy might be considered to prevent the recurrence of intussusceptions.

\section{Competing interests: None}

Endoscopy_UCTN_Code_TTT_1AP_2AD 
J.-H. Lai' ${ }^{1}$ C.-W. Chang', M.-J. Chen ${ }^{1,2}$, S.-C. Lin ${ }^{1}$, H.-Y. Wang ${ }^{1,2}$, C.-W. Chang ${ }^{1,2}$

1 Division of Gastroenterology, Department of Internal Medicine, Mackay Memorial Hospital, Taipei, Taiwan

2 Mackay Medicine, Nursing and Management College, Taipei, Taiwan

\section{References}

1 Ross AS, Dye C, Prachand VN. Laparoscopicassisted double-balloon enteroscopy for small-bowel polyp surveillance and treatment in patients with Peutz-Jeghers syndrome. Gastrointest Endosc 2006; 64: $984-$ 988

2 Kopacova M, Tacheci I, Rejchrt S, Bures J. Peutz-Jeghers syndrome: diagnostic and therapeutic approach. World J Gastroenterol 2009 Nov 21; 15: 5397-5408

3 Gao H, van Lier MG, Poley JW et al. Endoscopic therapy of small-bowel polyps by double-balloon enteroscopy in patients with Peutz-Jeghers syndrome. Gastrointest Endosc 2010; 71: 768 - 773

4 Tsujikawa T, Saitoh Y, Andoh A et al. Novel single-balloon enteroscopy for diagnosis and treatment of the small intestine: preliminary experiences. Endoscopy 2008; 40: $11-15$

\section{Bibliography}

DOI $10.1055 / \mathrm{s}-0030-1255809$

Endoscopy 2010; 42: E312 -E313

(c) Georg Thieme Verlag KG Stuttgart · New York . ISSN 0013-726X

\section{Corresponding author}

\section{C.-W. Chang, MD}

Division of Gastroenterology

Department of Internal Medicine

Mackay Memorial Hospital

No. 92, Sec. 2, Chung-Shan North Road

Taipei

Taiwan

Fax: +886-2-25433642

mky378@yahoo.com.tw 